\title{
High-Grade Chondrosarcoma Associated with Spindle Cell Components of the Cricoid Cartilage
}

\author{
Sohsuke Yamada ${ }^{{ }^{*}}$, Atsunori Nabeshima ${ }^{1}$, Hirotsugu Noguchi ${ }^{1}$, Aya Nawata $^{1}$, Hidetaka Uramoto ${ }^{2}$ and Toshiyuki Nakayama $^{1}$ \\ ${ }^{1}$ Department of Pathology and Cell Biology, School of Medicine, University of Occupational and Environmental Health, Kitakyushu, Japan \\ ${ }^{2}$ Department of Surgery, School of Medicine, University of Occupational and Environmental Health, Kitakyushu, Japan
}

*Corresponding author: Sohsuke Yamada, Department of Pathology and Cell Biology, School of Medicine, University of Occupational and Environmental Health, 1-1 Iseigaoka, Yahatanishi-ku, Kitakyushu 807-8555, Japan, Tel: 81-93-691-7426; E-mail: sousuke@med.uoeh-u.ac.jp

Rec date: Oct 09, 2014, Acc date: Nov 26, 2014, Pub date: Nov 28, 2014

Copyright: ( 2014 Yamada S, et al. This is an open-access article distributed under the terms of the Creative Commons Attribution License, which permits unrestricted use, distribution, and reproduction in any medium, provided the original author and source are credited.

\begin{abstract}
A history of recent frequency in cough and sputum was presented in a 73-year-old male. A neck CT scan revealed a heterogeneously enhanced and poorly-demarcated mass, measuring approximately $35 \times 35 \mathrm{~mm}$, arising from the right cricoid cartilage and projecting into the airway. The inadequate cytologic specimen contained few individual cells or small clusters of tumor cells having pleomorphic and sometimes spindle- or multi-nuclei, and abundant clear or vacuolated cytoplasm, along with an amount of metachromatic chondroid stroma. We first interpreted it merely as carcinoma. A total laryngectomy was performed, and gross examination showed a multilobulated and firm mass with a grayish to whitish, fleshy and glistening appearance, involving the cricoid cartilage and cricothyroid muscle. However, the overlying surface tracheal mucosa was smooth. Microscopically, the tumor was predominantly composed of a solid proliferation of atypical chondrocyte-like cells having hyperchromatic bi- or multi-nuclei in a lobular fashion, embedded in an abundant chondroid matrix, associated with cellular spindled cell foci. Therefore, we finally made a diagnosis of grade 2 chondrosarcoma with spindle cell components. We should be aware that owing to its characteristic features, cytopathologists might be able to determine correct diagnosis, based on multiple and adequate cytology samplings.
\end{abstract}

Keywords: Chondrosarcoma; Cricothyroid muscle; Spindled cell

\section{Introduction}

Among all laryngeal non-epithelial neoplasms, chondrosarcoma accounts for much less than $1 \%$ and, to date, more than 110 or up to 33 cases have only been reported in the English or Japanese literatures, respectively [1-4], whereas laryngeal chondrosarcoma arising from the cricoid cartilage constitutes more than $75 \%[1,2]$. This neoplasm often poses a diagnostic challenge to clinicians and cytopathologists, since it is frequently associated with a benign chondroma component and its entity is difficult to diagnose pre-operatively on an inadequate and small sample [1]. In fact, laryngeal chondrosarcomas can be asymptomatic before those patients present with airway stenosis or obstruction, as most of them are not widely invasive on gross findings [1-4]. However, patients even with high-grade chondrosarcoma could have a relatively better prognosis due to a much lower tendency to develop a recurrence or metastases [1,2]. Thus, early accurate diagnosis and conservative surgical treatment (i.e., voice-preserving surgery) can allow for an improved quality of life, as well as increase their survival rates [1]. Herein we reported an extremely rare case of high-grade chondrosarcoma coexisted with spindle cell components originated from the cricoid cartilage, giving rise to the genuine diagnostic difficulty on an inadequate cytology specimen.

\section{Case Report}

The patient presented here, a 73-year-old male with an unremarkable previous medical history, had a complaint of recent frequency in cough and sputum. Laboratory data, including blood cell count, chemistry and tumor markers, were within normal limits (carcinoembryonic antigen (CEA; $2.1 \mathrm{ng} / \mathrm{mL}$ ), carbohydrate antigen (CA) 19-9 (CA19-9; 8.3 U/mL), sialyl Lewis X-i antigen (SLX; $312 \mathrm{U} /$ $\mathrm{mL}$ ), squamous cell carcinoma antigen (SCC; $0.6 \mathrm{ng} / \mathrm{mL}$ ), cytokeratin 19 fragment (CYFRA; $1.6 \mathrm{ng} / \mathrm{mL}$ ), neuron specific enolase (SLE; 13.2 $\mathrm{ng} / \mathrm{mL}$ ), and pro-gastrin-releasing peptide (pro-GRP; $51.0 \mathrm{pg} / \mathrm{mL}$ )), and he displayed euthyroidism, i.e., free T3 and T4, thyroidstimulating hormone, and thyroglobulin was also within the normal range. A neck CT scan revealed a heterogeneously enhanced and poorly-demarcated mass with focal calcification, measuring approximately $35 \times 35 \mathrm{~mm}$ in diameter, arising possibly from the right cricoid cartilage or thyroid gland and projecting into the airway (Figure 1A). Full-body CT scans disclosed no definite evidence of metastases in the lymph nodes or other organs. Bronchoscopy showed a protruding submucosal tumor lesion at the right subglottic larynx, covered by smooth surface of tracheal mucosa. Clinicians first diagnosed it as a thyroid undifferentiated carcinoma or salivary gland type carcinomas. The inadequate specimen from the percutaneous fine needle aspiration (FNA) cytology contained few individual cells or small clusters of tumor cells having pleomorphic and sometimes spindle- or multi-nuclei, and abundant clear or vacuolated cytoplasm, along with a substantial amount of metachromatic chondroid stroma, without any evidence of necrotic or hemorrhagic backgrounds (Figure 1B). We cytopathologists interpreted it merely as carcinoma, including mucoepidermoid carcinoma as one of differential diagnoses. A total laryngectomy with combined resection of right thyroid gland was thus performed, and gross examination displayed a multi-lobulated and firm mass with a grayish to whitish, fleshy and glistening appearance, involving the cricoid cartilage and cricothyroid muscle, and compressing the pre-existing right thyroid gland (Figure 1C). On scanning magnification, the tumor showed a poorly-circumscribed, uncapsulated and basophilic cartilaginous mass lesion in a multi- 
Citation: Yamada S, Nabeshima A, Noguchi H, Nawata A, Uramoto H, et al. (2014) High-Grade Chondrosarcoma Associated with Spindle Cell Components of the Cricoid Cartilage. J Cytol Histol S4: 020. doi:10.4172/2157-7099.S4-020

Page 2 of 3

lobulated fashion, aggressively invading the cricoid cartilage and cricothyroid muscle, but not the thyroid gland or covering tracheal epithelium (Figure 1D). Resection was diagnosed as complete by this histopathological examination. The covering tracheal epithelium showed no remarkable change. Microscopically, its multi-lobulated parts were composed of a solid proliferation of atypical chondrocytelike cells with focal calcification and coagulative necrosis, embedded in an abundant and basophilic chondroid matrix, very likely originated from the mature-looking cricoid cartilage (Figure 2A). No classic "string of pearls" configuration by a myxoid stroma was identified [1]. On high-power view, these neoplastic cells revealed a small to medium-sized and round shape, having hyperchromatic bi- or multinuclei, inconspicuous nucleoli, abundant clear vacuolated cytoplasm, and indistinct cellular borders (Figure 2B). Mitotic figures were sometimes encountered. Moreover, these neoplastic lobules partly coexisted with cellular spindled cell foci (Figure 2C). Immunohistochemically, the atypical chondrocyte-like cells were strongly positive for S-100 protein (Dako Cytomation Co., Glostrup, Denmark, diluted 1:900), but the atypical spindle cells were not (Figure 2D). By contrast, both neoplastic components were specifically positive for Sox-9 (Santa Cruz Biotechnology, Santa Cruz, CA, USA; diluted 1:50). Based on all these features, we finally made a diagnosis of high-grade (grade 2) chondrosarcoma associated with spindle cell components arising from the cricoid cartilage. No additional malignant mesenchymal components were seen, and there were very few benign chondroma components, within our thorough investigation. To date, approximately 2 years routine follow-up after the surgery is established, and the patient remains well and no recurrence has been recognized.

\section{Discussion}

Aggressive clinical treatment in the early stage for highly invasive chondrosarcoma of the larynx might be the hope for better prognosis, due to a higher-grade (i.e., grade 3 or dedifferentiation), malignant tumor $[1,2,4]$, whereas the overall nature of cartilaginous tumors from the larynx appears generally indolent, except for a myxoid-type chondrosarcoma [1]. Thus, it is critical to establish an accurate preoperative diagnosis by FNA cytology/biopsy, the clinical utility of which in diagnosing neck tumors has been generalized [1,4-6]. However, findings regarding FNA cytology/biopsy for the laryngeal cartilaginous neoplasms have been very rarely reported, since these methods are not always successful due to the firmness of these tumors $[5,6]$. To the best of our knowledge, this is actually the first single-case report of high-grade chondrosarcoma with spindle cell components, especially focusing on its cytologic findings. The cytology could reflect the histopathological ones resembling not only chondroma to chondrosarcoma but possibly spindle cell tumor, showing individual chondrocyte-like cells or small clusters of tumor cells along with a substantial amount of metachromatic chondroid stroma, in the background of necrosis or hemorrhage $[5,6]$. As in the present case, although the specimens were inadequate, the cytologic features were almost similar to those as described above, even while relatively clear backgrounds were seen. Nevertheless, a confident and accurate diagnosis of high-grade chondrosarcoma with spindle cell components was impossible merely on cytology, due to sampling errors, lack of experience, cytomorphologic variety, and/or misinterpretation. Therefore, in cases with a strong clinical suspicion of laryngeal cartilaginous tumors, multiple and CT-guided, if possible, FNA cytology/biopsy should be performed and its suspicion must be raised to alert the cytopathologists, at the very least. Furthermore, an immunohistochemical analysis indicates that S-100 protein and/or Sox-9 are highly and specifically expressed in patients with low- to high-grade chondrosarcoma, respectively $[1,4]$, as described here. Indeed, we can propose that, when the cytologic features of highergrade chondrosarcoma with a variety of cell morphology, e.g., benign chondroma to dedifferentiated chondrosarcoma, are difficult to make an accurate and conclusive diagnosis [1], immunostaining for those 2 markers on cytologic smears or cell block preparations might be very useful for the exclusion from carcinoma, at least, and subsequently the correct interpretation of it. Despite that, future studies will be further required to determine whether our suggestion is significant after collecting and examining a larger number of its cases.

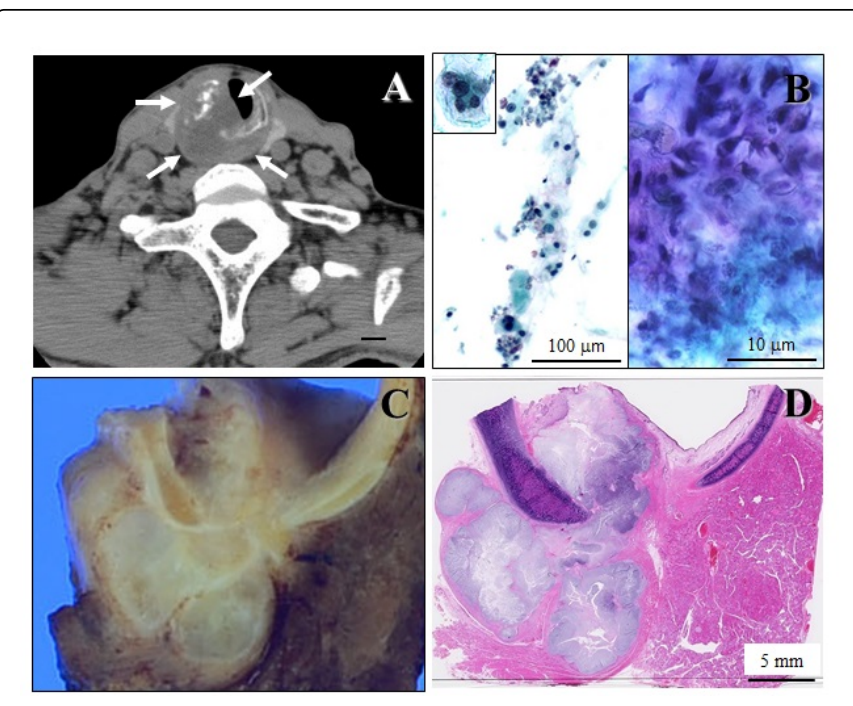

Figure 1: The findings of neck CT at surgery, or gross and microscopic examination of the resected high-grade chondrosarcoma specimen. (A) A full neck CT scan demonstrated a heterogeneously enhanced and poorly-demarcated mass (arrows) with focal calcification, measuring approximately $35 \times 35 \mathrm{~mm}$ in diameter, arising possibly from the right cricoid cartilage or thyroid gland and projecting into the airway. Bar $=1 \mathrm{~cm}$. (B) The inadequate specimen from the percutaneous FNA cytology contained few individual cells or small clusters of tumor cells having pleomorphic and sometimes spindle- or multi-nuclei (inset), and abundant clear or vacuolated cytoplasm (lt., Papanicolaou stains), along with a substantial amount of metachromatic chondroid stroma (rt., Giemsa stain), without any evidence of necrotic or hemorrhagic backgrounds (lt.). Bars $=100 \mu \mathrm{m}$ (lt.) and $10 \mu \mathrm{m}$ (rt.). (C)(D) Gross examination $(\mathrm{C})$ of the resected laryngeal chondrosarcoma showed a multi-lobulated and firm mass with a grayish to whitish, fleshy and glistening appearance, involving the cricoid cartilage and cricothyroid muscle, and compressing the pre-existing right thyroid gland (rt. side). On its scanning magnification (H\&E stains) (D), the tumor revealed a poorly-circumscribed, uncapsulated and basophilic cartilaginous mass lesion in a multi-lobulated fashion, aggressively invading the cricoid cartilage and cricothyroid muscle, but not the thyroid gland (rt. side) or covering tracheal epithelium (upper rt.). Bar $=5 \mathrm{~mm}$. 
Citation: Yamada S, Nabeshima A, Noguchi H, Nawata A, Uramoto H, et al. (2014) High-Grade Chondrosarcoma Associated with Spindle Cell Components of the Cricoid Cartilage. J Cytol Histol S4: 020. doi:10.4172/2157-7099.S4-020

Page 3 of 3

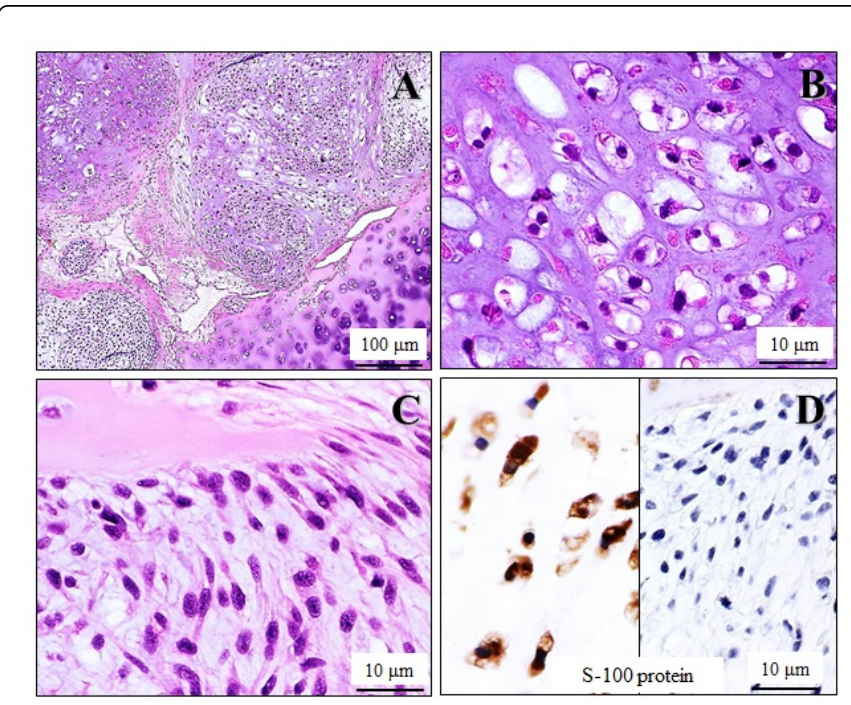

Figure 2: Microscopic and immunohistochemical examination of the high-grade chondrosarcoma with spindle cell components arising from the cricoid cartilage. (A) Low-power view (H\&E stains) showed that its multi-lobulated parts were composed of a solid proliferation of atypical chondrocyte-like cells, embedded in an abundant and basophilic chondroid matrix, very likely originated from the mature-looking cricoid cartilage (lower rt.). Bar $=100 \mu \mathrm{m}$. (B) High-power view demonstrated that these neoplastic cells had a small to medium-sized and round shape, displaying hyperchromatic bi- or multi-nuclei, inconspicuous nucleoli, abundant clear vacuolated cytoplasm, and indistinct cellular borders (H\&E stains). Bar=10 $\mu \mathrm{m}$. (C) These neoplastic lobules coexisted with cellular spindled cell foci in part. Bar $=10 \mu \mathrm{m}$. (D) In immunohistochemistry, the atypical chondrocyte-like cells were specifically positive for S-100 protein (lt.), but the atypical spindle cells were not (rt.) Bar=10 $\mu \mathrm{m}$.
We herein reported a case of a grade 2 chondrosarcoma with spindle cell components arising from the cricoid cartilage. The present case was tentatively misdiagnosed as carcinoma, NOS, on the examination of the cytology, following aggressive surgery, since its smears displayed the inadequate and few components of tumor cells. All cytopathologists should be aware that its clinicopathologically characteristic features, as well as multiple and adequate FNA specimens, could lead to a correct diagnosis.

\section{References}

1. Thompson LD, Gannon FH (2002) Chondrosarcoma of the larynx: a clinicopathologic study of 111 cases with a review of the literature. Am J Surg Pathol 26: 836-851.

2. Coca-Pelaz A, Rodrigo JP, Triantafyllou A, Hunt JL, Fernández-Miranda JC, et al. (2014) Chondrosarcomas of the head and neck. Eur Arch Otorhinolaryngol 271: 2601-2609.

3. Ohnishi M, Murai M, Jin H, Izuhara K, Kurotaki H, et al. (2009) A case of laryngeal chondrosarcoma. J Jpn Bronchoesophagol Soc 60: 336-343 (in Japanese with English abstract).

4. Mills SE, Stelow EB, Hunt JL (2012) Chondrosarcoma. In: Tumors of the Upper Aerodigestive Tract and Ear. Atlas of tumor pathology. Maryland: Armed Forces Institute of Pathology 1: 421-423.

5. Froberg MK, Meschter SC, Brown RE, Garbes AD (1996) Cartilaginous tumors of the larynx. A report of two cases with definitive diagnosis by fine needle aspiration and computed tomography. Acta Cytol 40: 761-764.

6. Ibarrola C, Vargas J, de Agustín P (1998) Laryngeal chondrosarcoma: fine needle aspiration (FNA) of an unusual tumour. Cytopathology 9: $130-134$.
This article was originally published in a special issue, entitled: "Histology and Histopathology", Edited by Borislav A. Alexiev, University of Maryland Medical Center, USA 